beaucoup de parties de la région et particulièrement dans la vallée d'Aspe? Pour épargner aux torrents de cette vallée les alternances de sécheresse et de crues qu'ils subissent il taudrait des massifs de forêts et de pâturages boisés étendus, reliant les uns aux autres beaucoup des bois isolés d'aujourd'hui, recouvrant ou interrompant la nudité de tant de versants déclives où rien n'arrête ni même ne retarde le ruissellement.

Ainsi rétablirait-on sur le sol l'éponge modératrice, le réservoir naturel, qui obvierait aux crues des gaves comme aux périodes de basses eaux et empêcherait leur débit de se réduire à l'excès.

Pierre Buffault, Inspecteur adjoint des Eaux et Forêts

\section{TRANSPORTEUR HYDRAULIQUE POUR LES BOIS DÉBITÉS}

Au cours d'un voyage en Roumanie, j'ai eu l'occasion de voir appliquer au transport des planches une installation hydraulique des plus simples et des plus pratiques.

Elle consiste en un canal en planches et ce système doit être fréquemment employé dans la région, ainsi qu'en Transylvanie. Il l'est peut-être aussi dans d'autres pays, peut-être même en France, mais je ne l'ai vu que dans les vallées de deux ruisseaux roumains, la Bicaz et le Tarcàn, à peu de distance de la frontière transylvane. Il convient aux longs parcours et aux faibles pentes - deux qualités précieuses évidemment pour un transporteur - et j'ai pensé que sa description pourrait intéresser les lecteurs de La Honille Blanche.

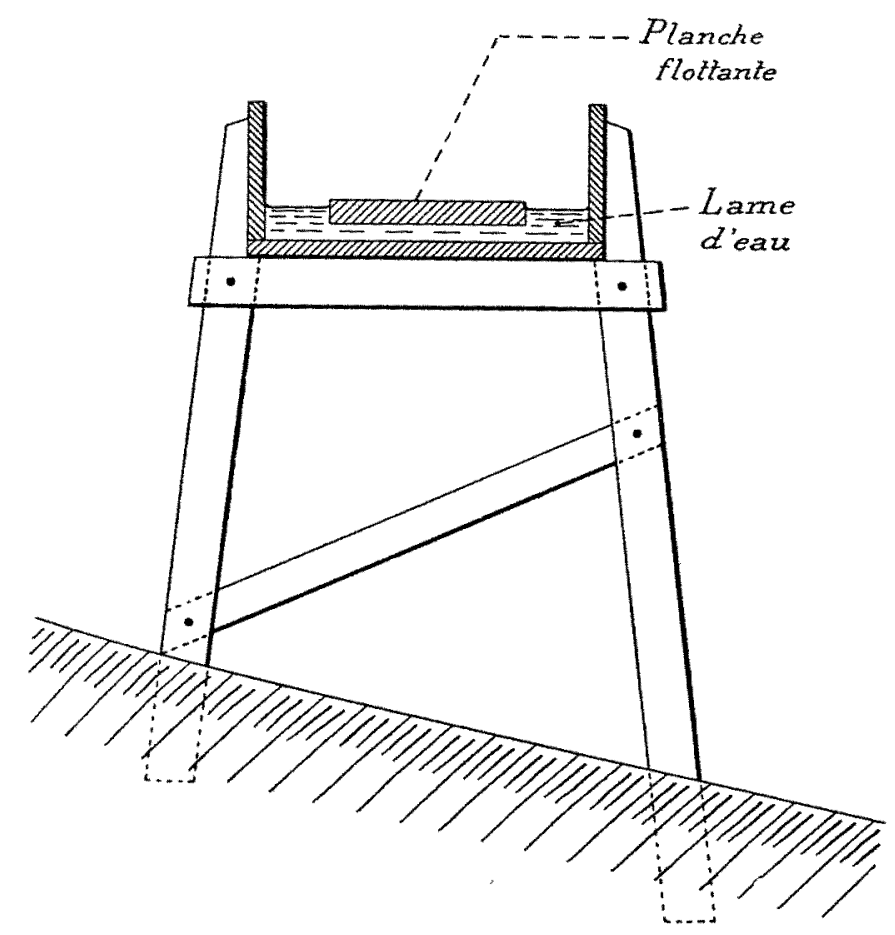

Cet appareil est donc un canal en planches, découvert, ayant environ 70 centimètres de largeur et 30 de profondeur; il suit la vallée, tantôt placé sur des chevalets, tantôt posé sur le sol, tantôt même partiellement enterré, de manière à offir une pente aussi régulière que possible et qui varie, me semble-t-il, entre 5 millimètres et 50 millimètres par mètre. Il présente des courbes, mais celles-ci sont, évidemment, d'un rayon aussi grand que le terrain le permet; enfin, la longueur atteint plusieurs kilomètres et permet de débiter les planches dans la forêt et de les amener à une gare de chemin de fer ou à un port fluvial.

C'est cette longueur qui frappe tout d'abord, lorsque l'on voit ce canal se développer à côté d'une route.

Quant au fonctionnement du système, le lecteur l'a déjà compris : on envoie de l'eau dans le canal; le régime s'établit bientôt et, sur la nappe liquide régulière, on abandonne les planches, une à une, comme autant de flotteurs destinés à mesurer la vitesse du courant, en ménageant entre eux un intervalle de plusieurs mètres; les planches gardent leurs distances et passent parfaitement dans les courbes.

La recette se fait de la manière suivante : le canal aboutit à un vaste terrain ou entrepôt de manière que son extrémité libre se trouve à plusieurs mètres au-dessus du sol; il est, pour cela, supporté par des chevalets de plus en plus élevés (nous supposons évidemment qu'aux abords du point choisi la pente du terrain est supérieure à celle donnée au canal). Les choses étant ainsi disposées, l'eau tombe en cascade à l'extrémité du canal et les planches tombent en même temps, mais elles vont plus loin et s'entassent assez régulièrement. Rien n'est curieux comme cette double cascade d'eau et de planches.

Il est évident que ce système pourrait s'appliquer à d'autres bois débités; mais alors il faudrait, en général, augmenter l'épaisseur de la lame d'eau, car c'est la planche qui offre, à volume égal, le moins de profondeur d'immersion. On conçoit aussi qu'on peut l'appliquer sur des longueurs presque indéfinies, à la condition que la vallée offre une légère pente et que l'on installe (ce qui est généralement possible) des arrivées d'eau pour compenser les pertes.

L. Bravet.

Ingénieur des Arts et Manufactures.

\section{POTEAUX EN CIMENT ARMÉ SYSTËME A. BOURGEAT}

Dans l'installation des usines hydro-électriques puissantes destinées à alimenter en énergie des centres nombreux et éloignés, la question des poteaux devant supporter les canalisations aériennes prend une importance capitale.

Jusqu'à présent le problème était résolu par l'emploi de poteaux en bois ou de poteaux métalliques. Ces derniers donnent évidemment toute satisfaction, mais leur prix grève trop lourdement les frais d'installation. Même en augmentant les portées jusqu'à 70 ou 80 mètres, le prix d'installation de la ligne est cinq ou six fois plus élevé qu'avec des poteaux en bois.

Quant à ceux-ci, on connaît tous leurs inconvénients. Le principal est le peu de durée. On ne peut guère compter qu'un poteau en bois, très soigneusement injecté, dure, dans nos climats, plus de 15 ans, et 5 ou 6 ans seulement après la pose d'une ligne, certains supports sont à remplacer. En sorte qu'une canalisation, mise en service depuis quelques années seulement, n'est jamais en état et ne laisse guère de répit à ceux qui sont chargés de l'entretenir. 
Il est bien difficile d'opérer les remplacements sans des interruptions de service, toujours ennuyeuses dans un réseau étendu alimentant des appareils d'applications très variées; et cela devient une impossibilité en cas de services publics, des éclairages de villes, par exemple.

Aussi a-t-on cherché depuis longtemps un type de poteau dont le prix soit comparable à celui du poteau en bois, mais qui rivalise pour la durée avec le poteau métallique. mailles de $22 \mathrm{~mm}$. en fil de $\mathrm{t} \mathrm{mm}$. de diamètre, enveloppant l'armature d'une façon invariable ;

50 Un mortier de ciment artificiel de première qualité, au dosage de $I / 3$ en poids de ciment et $2 / 3$ de sable grossier très maigre et bien lavé, formant remplissage jusqu'à l'âme en bois et recouvrant toute l'armature et le treillis de $2 \mathrm{~cm}$. au moins.

Le rôle de l'âme en bois est complexe; elle sert d'abord de support à l'armature métallique pour la fabrication et

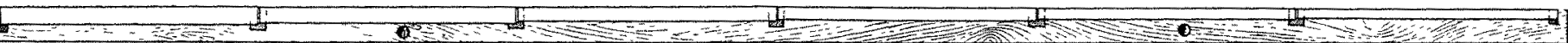

Moule servant à la confection des poteaux.

Dans le Dauphiné, pays du ciment, il était naturel de s'adresser à cet élément, dont l'emploi s'est si grandement étendu dans ces dernières années, par l'adjonction des carcasses métalliques dont on l'arme.

La Société Electro-Chimique de la Romanche, qui doit alimenter la ville de Grenoble avec ses usines de Livet, à 36 kilomètres de distance, vient d'adopter le poteau système Bourgeat de Voiron, breveté S. G. D. G. Il nous a paru que quelques détails à ce sujet pourraient intéresser les

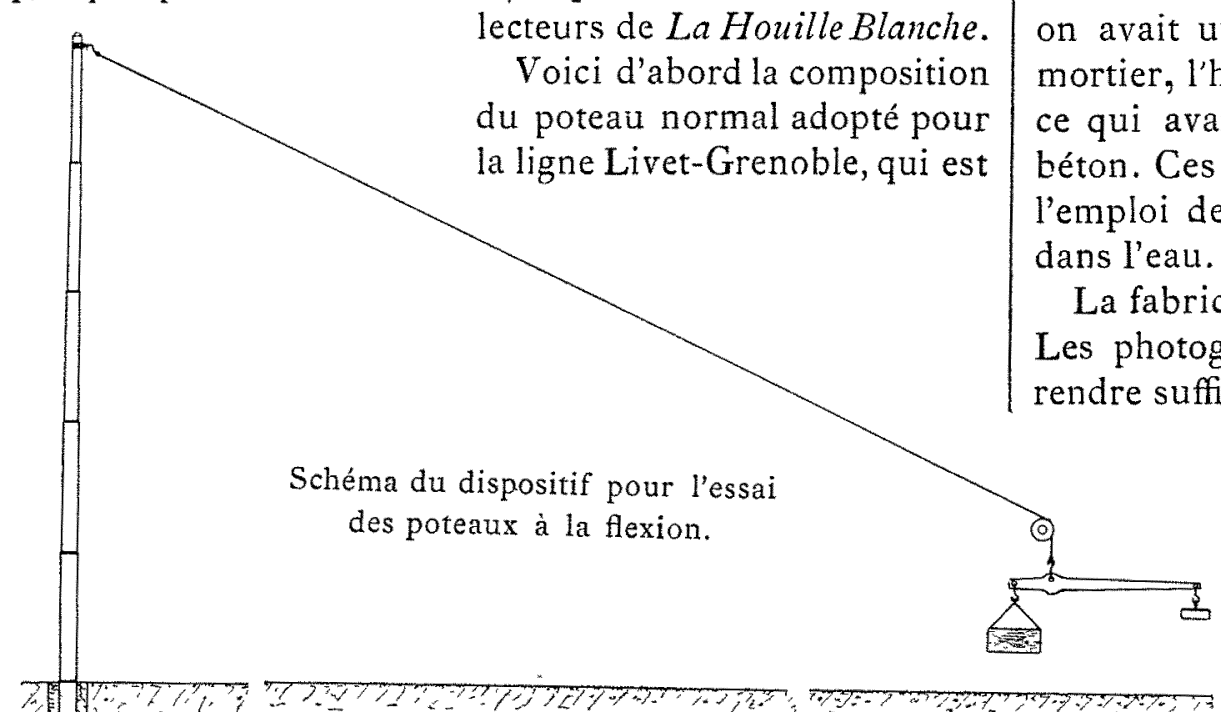
Voici d'abord la composition du poteau normal adopté pour la ligne Livet-Grenoble, qui est

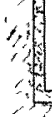

constituée par six fils de cuivre de $8 \mathrm{~mm}$. de diamètre, la tension variant entre 26.000 et 32.500 volts.

Longueur totale du poteau : I 2 mètres, dont I m. 80 encastré dans le sol, dans un massif de béton de chaux lourde de o m. 6 o de côté.

Le poteau est formé de :

$\mathrm{I}^{\circ}$ Une âme de sapin en grume, de o $\mathrm{m}$. I9 de diamètre à la base et o m. og au sommet;

$2^{\circ}$ Une hélice en fil ou câble de fer de $6 \mathrm{~mm}$. entourant l'âme, destinée à isoler l'armature de l'âme en bois pour qu'elle soit bien enrobée dans le ciment;

$3^{\circ}$ Huit tiges équidistantes en acier rond de $7 \mathrm{~mm}$. de diamètre, reposant sur l'hélice précédente, dans la position des génératrices d'un cône concentrique à l'âme en sapin, fortement tendues et reliées entre elles aux extrémités de l'âme ;

$4^{\circ}$ Un grillage en métal déployé ou toile métallique à en second lieu de moule intérieur. Une fois le poteau fabriqué, lâme en bois lui donne une certaine rigidité et permet de le démouler, de le transporter et de le manipuler de suite, c'est-à-dire une heure après le moulage. On ne compte guère, par la suite, sur la résistance du bois dans le calcul du poteau. Néanmoins ce bois étant tenu absolu. ment à l'abri du contact de l'air, pourra, pense-t-on, se conserver très longtemps.

Le bois employé doit être vert. Dans de premiers essais, on avait utilisé du bois sec. Mais, après l'application du mortier, l'humidité de ce dernier avait fait gonfler le bois, ce qui avait provoqué des fissures longitudinales dans le béton. Ces inconvénients ont été complètement évités par l'emploi de bois vert, ou de bois ayant trempé huit jours dans l'eau.

La fabrication de l'âme n'offre aucune difficulté spéciale. Les photographies jointes à cet article permettent de s'en rendre suffisamment compte.

Pour la confection du poteau, on se sert d'un moule métallique, représenté ci-dessus. Ce moule est composé d'une série de demicylindres ayant chacun 2 mètres de long et dont les diamètres diminuent de $2 \mathrm{~cm}$. de l'un à l'autre. Les poteaux ainsi obtenus ont un aspect télescopique.

Le moule est garni de mortier dans lequel on applique l'âme par une série de balancements. On la recouvre ensuite elle-même de mortier. Des demi-colliers se rabattent sur les points de variation de diamètre et les parties cylindriques sont ensuite travaillées à l'aide d'un lissoir ayant la forme d'un demi tronc de cône qui permet de comprimer le ciment en le moulant. L'extrémité supé* rieure du poteau, en forme de pointe, est obtenue par un moule conique spécial; l'extrémité inférieure, par une rondelle de bois.

Le ciment employé varie suivant la température. Par $20^{\circ}$, on employait, par exemple, moitié de ciment prompt ( 5 minutes de prise) et moitié de ciment lent ( 15 à 20 minutes de prise); mais la proportion de ciment prompt diminue avec l'élévation de la température pour disparaitre complètement vers $35^{\circ}$.

Le poteau peut être démoulé une demi-heure après la fabrication. Il peut alors être manipulé et transporté sans danger. Il possède d'ailleurs une grande élasticité, et au transport, on le voit fléchir notablement sans que la moindre fissure se produise. 
Ces poteaux peuvent être dressés huit à dix jours après leur fabrication ; aussi peuvent-ils être fabriqués sur place, ou du moins de distance en distance le long de la par sections télescopiques de 2 mètres, permet d'obtenir facilement des poteaux prolongés, qui sont indispensables en certains points bas de la ligne ou bien à la traversée d'autres

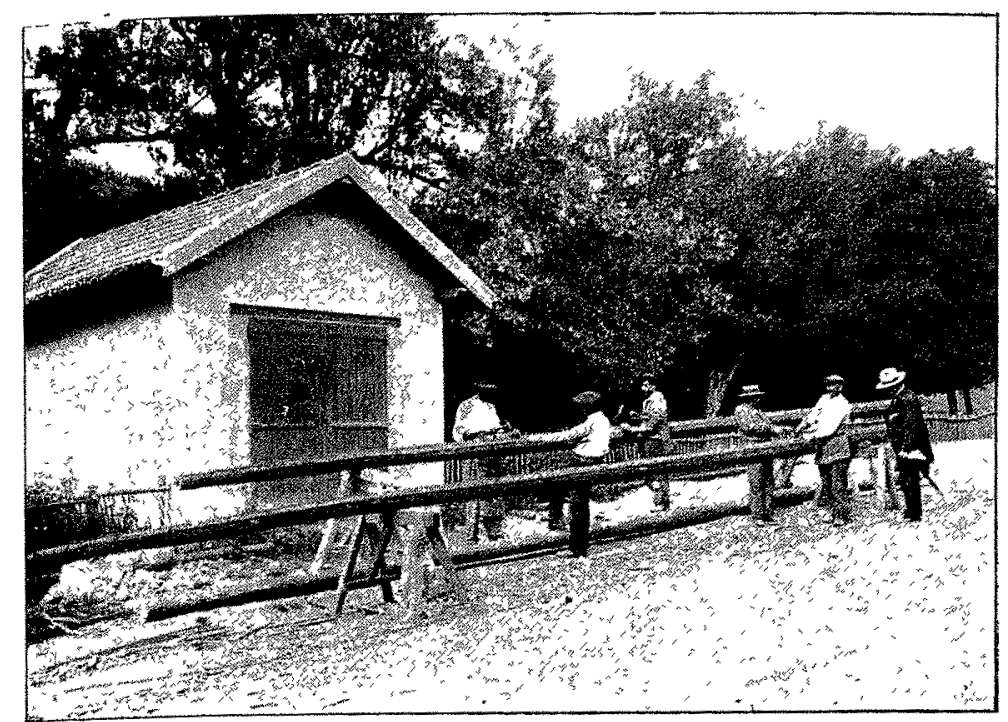

Placement de l'enveloppe en toile métallıque.

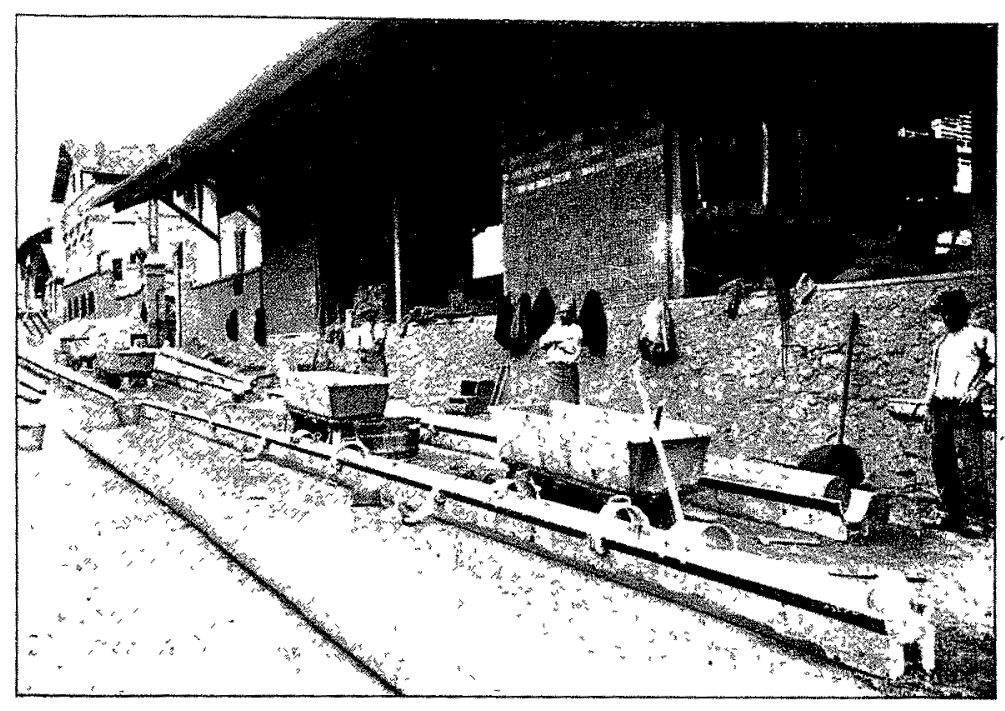

Moule, colliers, accessoires. ligne à installer. Nos photographies montrent les diverses phases de la fabrication dans un chantier installé à Eybens pour la première section de la ligne reliant Grenoble à Livet. Une équipe de 6 hommes, fabrique environ 15 poteaux par jour, et il entre dans la composition de chaque poteau à peu près 200 kilos de ciment. Le poteau terminé pèse approximativement 650 à 700 kilos.

Le système adopté pour la fabrication des poteaux,

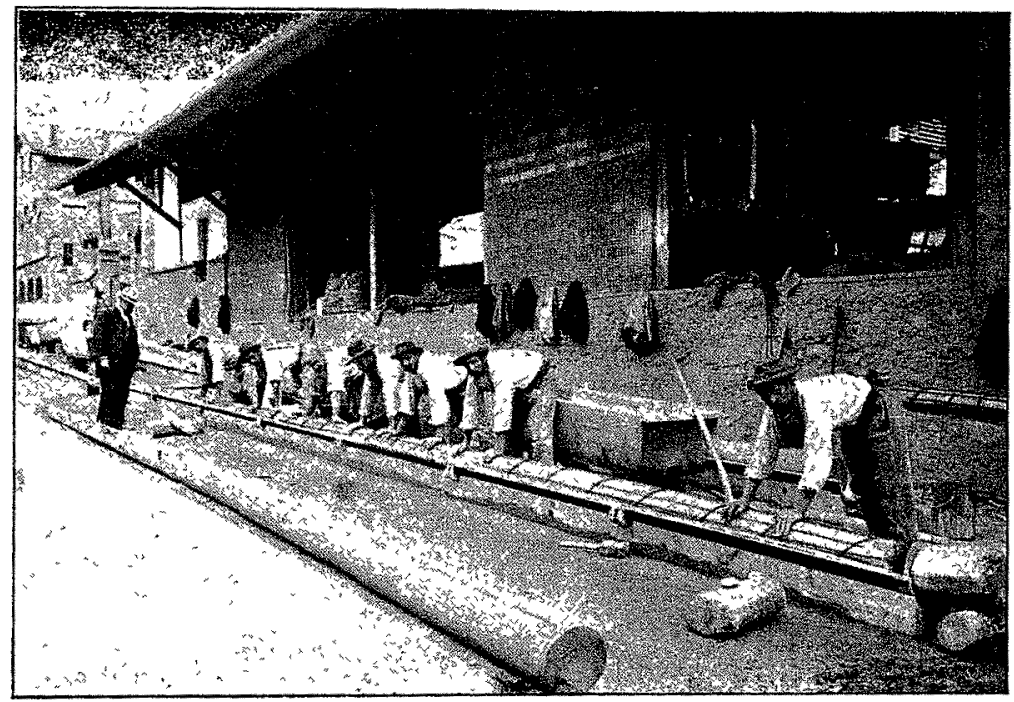

Placement de lâme dans le moule. canalisations électriques : télégraphe, téléphone, lumière, courant pour moteurs, etc.

On peut aussi farre des poteaux d'un diamètre plus fort, offrant une résistance beaucoup plus grande à la flexion, soit pour les points angulaires de la ligne, solt pour les points où les canalisations seraient plus considérables. C'est ainsi que toute la partie de la ligne située entre Livet et le Pont-de-Gavet ( 8 kilometres environ) sera constituée avec des?poteaux ren-

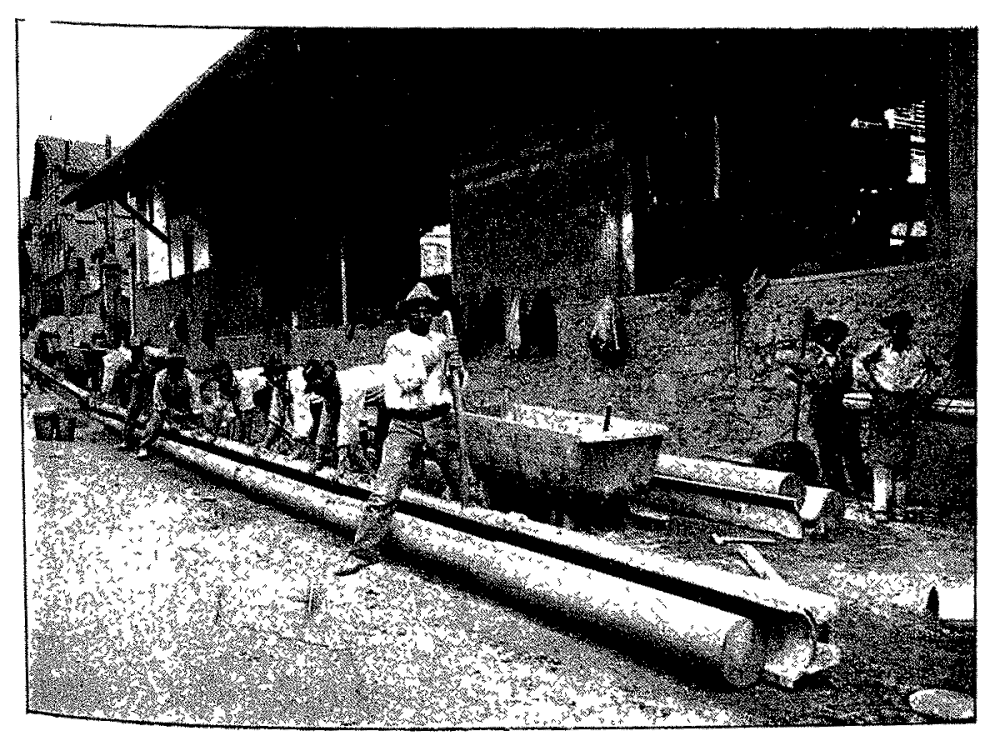

Démoulage d'un poteau.

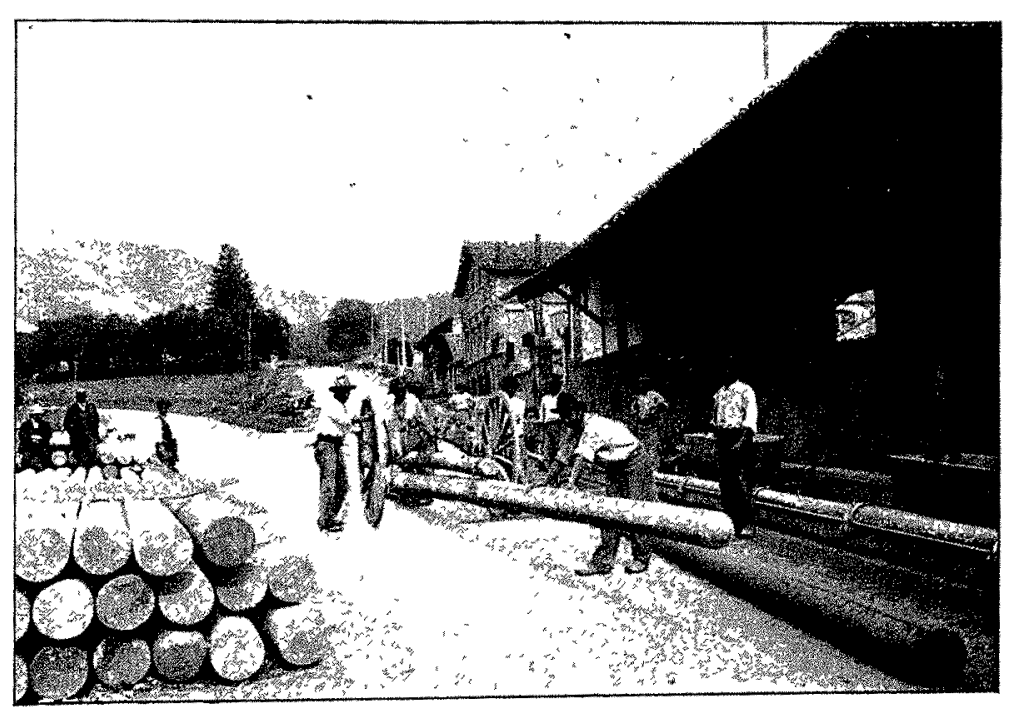

Transport d'un poteau, 
forcés ayant $5 \mathrm{~cm}$. de plus de diamètre. Sur ce parcours, la canalisation comporte 3 fils supplémentaires destinés à amener du courant de secours à l'usine d'Avigonnet de la Société Grenobloise de Force et Lumière.

L'âme métallique peut être facilement mise à la terre sans qu'aucun fil conducteur soit à l'extérieur et par conséquent à portée du public.

De même, on peut prolonger à la partie supérieure une des tiges de l'armature pour former paratonnerre.
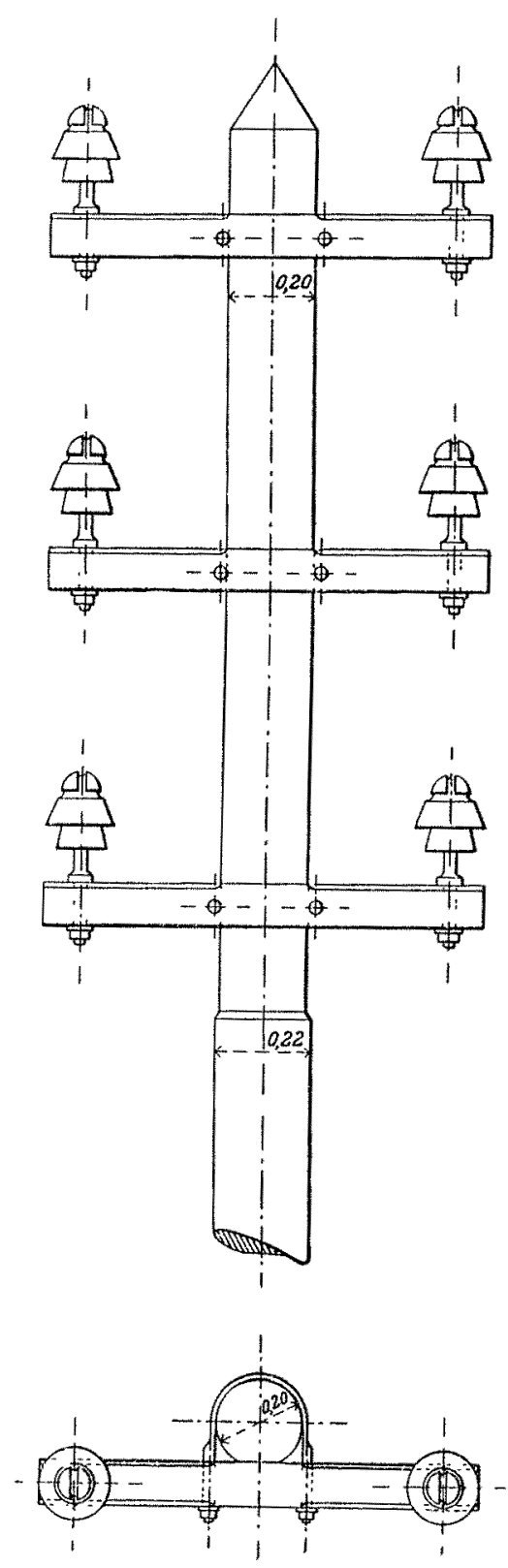

Poteau renforcé. par choc, la charge s'étant accrue brusquement de 50 kilogrammes tombant de om 30 environ.

Dans le second essai, on a chargé progressivement, en évitant tout choc, des poids servant à faire la charge. De plus, après chaque augmentation de charge, la flèche était mesurée, puis, en enlevant la charge totale, on vérifiait que le poteau reprenait bien sa position primitive. Le poteau est ansi revenu à la position verticale pour toutes les charges, sauf celle qui a provoqué la rupture.
Voici les chiffres trouvés :

\begin{tabular}{|c|c|c|c|}
\hline $\begin{array}{l}\text { Charge } \\
3 \text { I } 3 \mathrm{ks}\end{array}$ & $\begin{array}{l}\text { Hauteur de l'attache } \\
5 \mathrm{~m}_{9} 8\end{array}$ & $\begin{array}{l}\text { Flèche } \\
\text { o }^{\text {m }} 9\end{array}$ & $\begin{array}{l}\text { Rras de levier } \\
5 \mathrm{~m} 86\end{array}$ \\
\hline 367 & 》 & $\mathrm{o}_{24}$ & ) \\
\hline 404 & ) & $\mathrm{o}^{\mathrm{m}_{2}}$ & ") \\
\hline $47^{\circ}$ & ") & $o^{m_{2}} 7^{5}$ & D \\
\hline 500 & ") & $\mathrm{o}^{\mathrm{m}} 29 \mathrm{o}$ & ") \\
\hline 565 & " & Rupture & ) \\
\hline
\end{tabular}

Le moment de rupture a été de 3.300 kilogrammes. mètres; cette rupture s'est produite par écrasement du ciment en un point où celui-ci était défectueux. Pour toutes les charges, à part celle de rupture, la déformation était élastique et disparaissait quand on déchargeait le poteau.

Ces résultats demanderont évidemment à être contrôlés sur un grand nombre de poteaux et à recevoir la confirmation du temps.

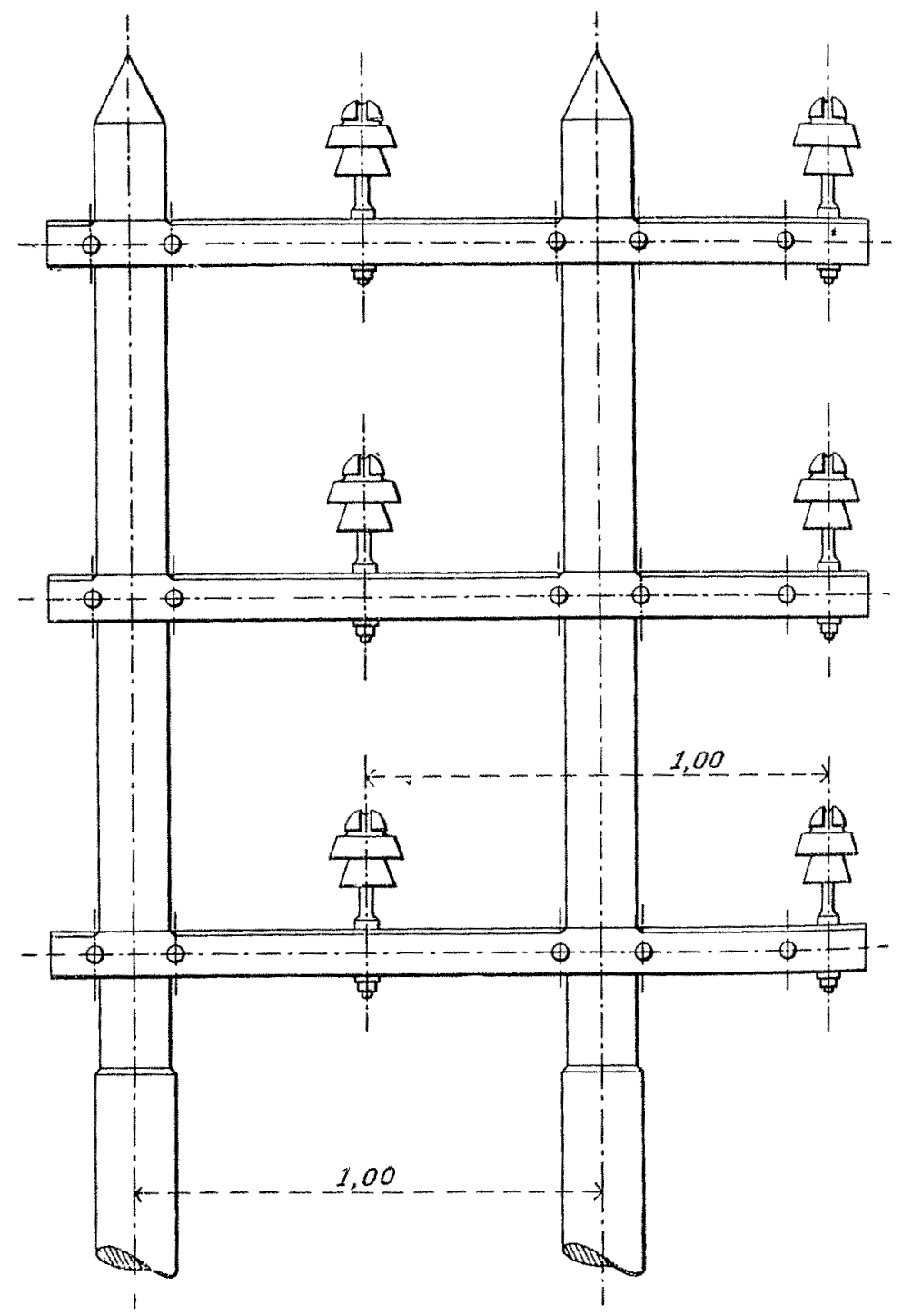

Poteaux accouplés.

Ajoutons, pour terminer, que le prix des poteaux en ciment, tout posés et fondés dans des blocs de béton, est pour le type de $\mathbf{2} 2$ mètres de longueur, de 25 à $35 \%$ supérieur au prix des poteaux en bois de même longueur ayant $\mathrm{o}_{2} 8$ à $\mathrm{o}^{\mathrm{m}} 3 \mathrm{o}$ de diamètre à la base.

Enfin les poteaux en ciment peuvent facilement recevoir un soubassement et une corniche leur donnant un aspect architectural au moins équivalent à celui des poteaux en tubes métalliques. La ville de Grenoble, qui avait interdit 
sur son territoire, l'emploi des poteaux en bois, par raison d'esthétique, a admis les poteaux en ciment.

Il est impossible de grimper sur les poteaux en ciment avec les appareils à pointes qui servent d'ordinaire avec les poteaux en bois; ou du moins, si cette ascension reste possible, il faudrait remplacer les pointes presque à chaque opération. D'ailleurs l'emploi des pointes n'est pas sans inconvénient pour les poteaux en bois; les piqûres produites sont un des éléments de détérioration les plus actifs du poteau, et il vaudrait encore mieux user les appareils qui servent à grimper que les poteaux eux-mêmes, et, comme nous venons de le dire, c'est ce qui arriverait avec les poteaux en ciment.

L'administration des Télégraphes, pour éviter la détérioration de ses poteaux par les pointes, a prescrit l'emploi, pour grimper aux poteaux, d'un appareil où le point d'appui obtenu par les pointes est remplacé par l'appui d'une corde enroulée autour du poteau. Ce nouveau système a été essayé sur les poteaux en ciment et y est parfaitement applicable.

La difficulté de monter aux poteaux en ciment sans appareil spécial est même une sécurité de plus contre les accidents dus à ceux qui, par malveillance ou par jeu, tentent l'escalade des poteaux en bois et surtout des poteaux en treillis.

Louis BRunhes,

Ancien Elève de l'Ecole Polytechnique,

Ingénieur à la Société Electro-Chimique de la Romanche.

\section{Messupe du rendement d'une djnamo de grande puissance (1)}

DE LAPPLICATION PRATIQUE DE LA

MÉTHODE DITE “PAR DÉCROISSANCE DE VITESSES »

La détermination du rendement d'une dynamo de grande puissance n'est pas une opération bien facile, surtout lorsque cette détermination se fait lors des essais de réception, la dynamo étant mise en place et accouplée avec son moteur.

Parmi toutes les méthodes connues, celles dites par pertes séparées sont à peu près seules applicables. L'une d'entre elles, fort ingénieuse, est susceptible, malgré de grandes difficultés, d'une application pratique. C'est la méthode dite " par décroissance de vitesse ». Nous rappelons en quelques lignes sa théorie fondée sur la discussion d'une équation de mécanique très simple.

Théorie. - On sait qu'il y a une relation entre le couple $C$ auquel est soumis un corps tournant, à chaque instant, et l'accélération angulaire $\alpha$. Cette relation est de la forme très simple : $\quad C=M \alpha$.

$M$ est le moment d'inertie $=\int m d^{2}$.

La puissance $P$ s'écrit en fonction du couple : $P=C_{\omega}$.

(1) Suite de l'étude sur : Les essais industriels des groupes hydroélectriques de grande puissance. Voir La Houille Blanche, no de mars 1903.
En remplaçant $C$ par sa valeur (I), il vient : $P=M_{\alpha(\omega)}$. Or, $\quad \alpha=\frac{d \omega}{d t} \quad$ d'où : $\quad P=M \omega \frac{d \omega}{d t}$

Considérons maintenant la masse tournante de la dynamo animée d'un mouvement de rotation. Si nous supprimons brusquement l'agent moteur, la vitesse va décroître suivant une certaine loi, et nous pourrons tracer la courbe de décroissance en portant en abscisses les temps et en ordonnées les valeurs de $\omega$ (fig. I). Cette courbe ne sera pas autre chose que la représentation graphique de l'expression algébrique de $\omega$ par rapport au temps.
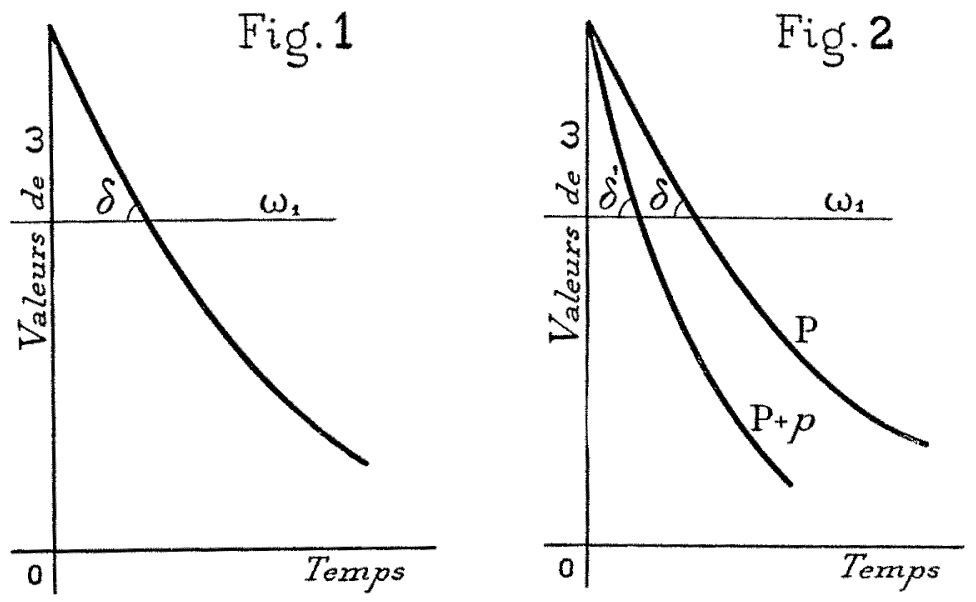

Considérons sur cette courbe l'ordonnée ( $\omega_{1}$ égale à la vitesse angulaire normale de la machine. A ce moment le couple résistant a une valeur $C$ telle que :

$C \omega_{1}=$ puissance dépensée par les résistances passives de la masse tournante.

Nous savons que cette puissance a pour expression:

$$
P=M_{\omega_{1}} \frac{d \omega}{d t}
$$

Or $\frac{d \omega}{d t}$ est la dérivée de l'expression de (1) par rapport au temps et cette dérivée nous la possédons car elle est égale à la tangente de l'angle que fait la courbe à cet endroit avec l'axe des temps; nous pouvons donc écrire :

$$
P=M_{\omega_{1}} \operatorname{tg} \delta
$$

Nous ne connaissons pas $M$ dont le calcul est presque impossible mais nous pouvons l'éliminer.

Pour cela ajoutons une résistance passive connue, un frein par exemple, dépensant à la vitesse (w) considérée une puissance $p$. Lançons de nouveau la masse tournante et traçons une nouvelle courbe de décroissance qui sera moins inclinée sur l'axe des temps (fig. 2). A la vitesse normale (1) la tangente à la courbe sera : Tg $\delta$ '.

et nous écrirons : $\quad P+p=M \omega_{1} \operatorname{tg} \delta^{\prime}$

d'où l'on tire :

$$
\frac{P}{P+p}=\frac{\operatorname{tg} \delta}{\operatorname{tg} \delta^{\prime}} \quad \text { et } \quad P=p \frac{\operatorname{tg} \delta^{\prime}}{\operatorname{tg} \delta^{\prime}-\operatorname{tg} \delta}
$$

Pour déterminer la perte dans le fer il suffira d'exciter convenablement la dynamo et de tracer une troisième courbe, On ajoutera, par cette excitation, une résistance passive $f$ correspondant à la puissance dépensée dans le fer. On me- 